\title{
HIGH ACCURACY ASTROMETRY BENEFITS FOR CELESTIAL MECHANICS
}

\author{
P. K. SEIDELMANN \\ U. S. Naval Observatory \\ Washington, D.C.
}

\begin{abstract}
.
Achieving angular accuracies at milli- and microarcsecond levels for objects within the solar system presents real challenges concerning the meaning of the observations due to the effects of phase and due to the sizes of the objects being observed. The use of retroreflectors or transmitters located on solar system objects may be the most promising technique.

However, if these accurate observations can be achieved, this provides the opportunity for significant improvements in the orbits of the planets, satellites, and minor planets, and will provide, or require, improvements in the models for the tidal interactions, secular accelerations, and masses. The observations will provide a test, or improvement, of the ties between radar, optical, and radio observations. This should provide an opportunity for improving the reductions of pulsar timing observations and improve our determination of the solar system reference system. This will also test the accuracy of the equivalence between Atomic Time and Dynamical Time. These accuracy levels should permit the discovery of other solar systems and the resulting increase in our knowledge, based on more than a single solar system.

Improvements in the accuracy level of observations provide increases in our knowledge and raises new challenges to explain the signatures detected by the observations.
\end{abstract}

\section{Introduction}

For solar system objects, there is a continuing interaction between observational accuracy and theoretical improvements. In principle, the method of computing the orbit, or the theoretical orbit computations, should be an. 
order of magnitude more accurate than the observational data. Once the observations become more accurate, it requires improved methods of orbit computation or it reveals the errors in the previous method. So, there is a continuing cycle of observational improvement, computational improvement, observational improvement, etc. Thus, as we contemplate observational improvement, we can anticipate the improvement in theory and the possibilities of new effects which must be included in our models of the solar system.

\section{Observational Challenge}

The benefits to celestial mechanics of milli- to microarcsecond angular astrometry will come primarily from observations of solar system bodies to that accuracy level. However, achieving optical angular accuracies will be a challenge. The solar system bodies present a very different image than a stellar point source. The solar system bodies are extended sources. They have phase effects. Thus the Navy Prototype Optical Interferometer has the problem that, if the objects are bright enough, then they are also large enough that the interferometer will resolve the body. Larger siderostats would permit unresolved observations of small minor planets.

The highly accurate observations of the solar system today are not made by angular measures. The high accurate observations are lunar laser ranging, radar ranging, radar illumination and VLBI measurement, millisecond pulsar recordings, spacecraft, and mutual events; specifically occultations, eclipses, or objects observed when they are very close to each other, being equivalent to a mutual event. Since one milliarcsecond at one AU is $0.7 \mathrm{~km}$ and one microarcsecond at one $\mathrm{AU}$ is $0.7 \mathrm{~m}$, radar to inner planets is at mas level, some spacecraft are at $10 \mu$ as and lunar laser ranging is at $50 \mu$ as level for some parameters.

What are the future possibilities for accurate observations? The ones that come to mind are putting a transponder on a solar system body, or placing a small emitting object in orbit around the planet. Alternatively, can we come up with a new technique? Accurate observations of selected asteroids when passing near other asteroids would provide a means of determining the mass of the asteroid. This is essential to improving the model of the solar system for computations of improved ephemerides. This would, with the diameter, provide density and composition information about the asteroid.

\section{Theoretical Challenges}

As mentioned, improvements in observations require improvements in orbit computational methods. Some of the limitations on the theoretical methods 
are recognized and known. The current computation of the ephemerides does not include a complete model of the asteroid belt, or alternatively, all the individual asteroids. Likewise, the Kuiper belt and the Oort cloud are not included in the present model. Galactic effects are not included in the computed orbits as a direct effect. The tidal interactions between the extended bodies are not included, except in the Earth/Moon case. Improved accuracies will require higher order relativistic terms to be included in the equations of motion, or post-post-Newtonian approximations.

\section{Observational Benefits}

What are the benefits of more accurate observations? It should be stated here that individual, more accurate, observations will not be of any real benefit to the solar system. To achieve benefits it is necessary to have a continuing supply of more accurate observations in a systematic manner. With this type of observation, more accurate orbits can be determined, and in turn, more accurate astronomical constants. Assuming these observations are of different types, then more accurate ties between radio, radar, or optical frames can be determined. The secular accelerations can be determined, in addition to those for the Moon, Phobos, and Deimos. Improved values of the masses of satellites and asteroids can be determined. Improved orbits of the asteroids permit better determination of the families of the asteroids. With the improved ephemerides of the solar system, the millisecond pulsar observations can be more accurately reduced and the potential for a long-term time scale is improved. Also, the improved orbit determinations provide a capability of determining the dynamical time scale more accurately, and to test the equivalence between the atomic and dynamical time scales.

In time, these more accurate orbits may permit us to determine the changes taking place in the solar system, and the status of the evolution of the solar system. However, the capabilities in trying to trace back the solar system should be limited due to chaos.

More accurate observations will provide the astrometric observations of motions of stars, for studies of planets around other stars. This should provide information about other solar systems besides our own, which in turn should lead to knowledge about how solar systems are formed and the characteristics of other solar systems.

Finally, as we achieve more accurate observations, this should lead to the discovery of other phenomena. Systematic differences between the observations and the computed ephemerides have in the past, and should, in the future, indicate the causes of these discrepancies, and new phenomena. 


\section{Conclusion}

Improved observational accuracies for solar system bodies will be much more difficult than for stars. However, if the improvements can be achieved, they will require improvements in the theoretical methods for computing orbits. This, in turn, provides benefits in improved accuracy and new knowledge based on the improvement in accuracy of both the observations and the computations. 